\title{
On the outburst of symbiotic stars
}

\author{
H. Nussbaumer and M. Vogel \\ Institute of Astronomy \\ ETH Zentrum \\ 8092 Zürich (Switzerland)
}

SUMMARY. The most commonly advocated models for explaining the outburst of symbiotic systems are thermonuclear runaways, modulated mass accretion through a disc, or accretion disc instabilities. We show that the onset of a strong stellar wind on the cool component can also produce the observed increase in luminosity.

\section{The proposed mechanism}

In 1983 Nussbaumer and Schmutz published a study on HBV 475 ( $=\mathrm{V} 1329 \mathrm{Cyg}$ ) in which they suggested, as one of several possibilities, that the nebula observed in symbiotic systems may actually be the wind of the cool star, ionized by the radiation of the hot component. Nussbaumer and Vogel (1987) have carried that idea through a properly calculated model. As a qualitative new idea they propose that the outburst of symbiotic systems can also be explained by the onset of a strong stellar wind on the cool component. The growing wind presents an increasing target for the ionizing photons. A wind of $20 \mathrm{~km} / \mathrm{s}$ creates within one year a target with a radius of $\approx 6 \cdot 10^{13} \mathrm{~cm}$, which is probably a typical distance for the binary separation in symbiotic systems. The outburst of symbiotic stars occurs typically over a period of several months, up to well over one year. There are two critical questions:

(1) How likely is the simultaneous presence of an M-giant in a state of heavy mass-loss, and a hot component with a radiation temperature of $T^{*} \gtrsim 10^{5} \mathrm{~K}$.

(2) What are the outburst magnitudes, $\Delta M$, that can be reached.

White dwarfs are candidates for hot components. Their lifetimes at $T^{*}>10^{5} \mathrm{~K}$, however, is only about 100000 years (Koester and Schönberner, 1986). The simultaneous presence of a hot white dwarf and a red giant with massive mass-loss would therefore be best realised in double star systems where both components are of about equal mass. Their evolutionary times would therefore be about equal. 
Figure 1.

Luminosity variation in the B-band as a function of mass-loss rate. The dashed line shows the contribution of the nebular continuum to the total luminosity. The Figure does not include the contribution of the cool star. For mass loss rates $\gtrsim 10^{-6} \mathrm{M}_{\odot} \mathrm{yr}^{-1}$ the luminosity in the emission lines is reduced due to collisional de-excitation.

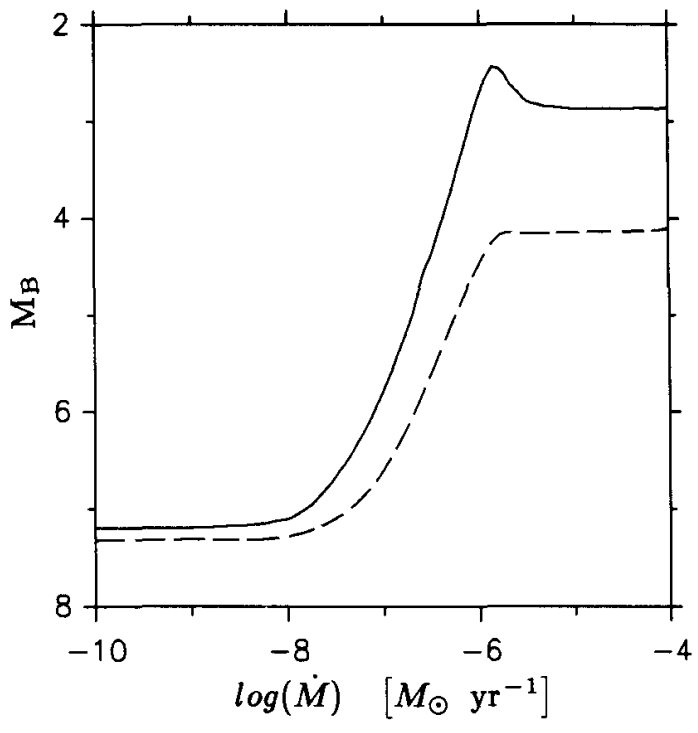

In Fig.1 we show the luminosity increase in the B-band due to the onset of a strong stellar wind. The model assumes the following physical conditions: A hot star with radius $R^{*}=$ $9 \cdot 10^{8} \mathrm{~cm}$ radiates as a blackbody with radiation temperature $T^{*}=1.5 \cdot 10^{5} \mathrm{~K}$. At a distance $p=5 \cdot 10^{13} \mathrm{~cm}$ a cool star is losing mass through a wind of the form

$$
\dot{M}=4 \pi r^{2} \mu m_{\mathrm{H}} N(r) v_{\infty}\left(1-\frac{R}{r}\right)
$$

The mean molecular weight is taken to be $\mu=1.4$, the terminal velocity is $v_{\infty}=80 \mathrm{~km} / \mathrm{s}$ and the origin of the stellar wind lies at $R=50 R_{\odot} . N(r)$ is the hydrogen density by number, $r$ is the distance from the center of the cool star and $m_{\mathrm{H}}$ is the mass of the hydrogen atom.

\section{Conclusions}

The outburst-model presented here is not meant to be exclusive. Thermonuclear runaways and unstable disc accretion are theoretically viable candidates for producing the observed outbursts. However, the symbiotic phenomenon may not be due to a unique event, valid in every system. Our wind outburst model has the right properties to match observations. We are not aware of any observational or theoretical objections excluding the possibility of a rapid onset of massive mass-loss through a wind in red giants.

Acknowledgments: M. Vogel thanks the Swiss Science Foundation and the Swiss Society of Astronomy and Astrophysics for financial support.

\section{References:}

Koester, D., Schönberner, D.: 1986, Astron.Astrophys. 154, 125

Nussbaumer, H., Schmutz, W.: 1983, Astron.Astrophys. 126, 59

Nussbaumer, H., Vogel, M.: 1987, Astron.Astrophys. 182, 51 